\title{
Contemporary Guidelines for Tympanostomy Tube Placement
}

\author{
Bianca Siegel, $M D^{1,2}$ \\ David H. Chi, MD ${ }^{1,2, *}$

\section{Address} \\ ${ }^{1}$ Children's Hospital of Pittsburgh of UPMC, Pittsburgh, PA, USA \\ *,2University of Pittsburgh Medical Center, Pittsburgh, PA, USA \\ Email: David.chi@chp.edu
}

Published online: 24 July 2015

(C) Springer International Publishing AG 2015

This article is part of the Topical Collection on Otolaryngology

Keywords Tympanostomy tube $\cdot$ Otitis media with effusion - Recurrent otitis media · Audiogram · Adenoidectomy

\section{Opinion statement}

Although tympanostomy tube placement has been one of the most common surgical procedures in children in the USA for many years, clinical practice guidelines were not developed until recently. In 2013, the American Academy of Otolaryngology-Head and Neck Surgery (AAO-HNS) published a clinical practice guideline based on an extensive literature review to address specific indications for surgery. These clinical practice guidelines focus primarily on chronic otitis media with effusion and recurrent acute otitis media and provide an evidence-based framework for decision-making strategies. Although a useful tool to assist in clinical decision-making, it is important to keep in mind that individual patient considerations and circumstances must also be taken into account when making treatment decisions. Otherwise, healthy children with minimal risk of developmental delays should be given a generous observation period prior to offering tympanostomy tube placement; higher-risk children should undergo more prompt surgical intervention.

\section{Introduction}

Tympanostomy tube placement is one of the most common pediatric surgical procedures performed in the USA $[1 \bullet \bullet]$. The most common indications are chronic otitis media with effusion (OME) with associated hearing loss and recurrent acute otitis media (RAOM). Other indications include complications of eustachian tube dysfunction, complications of acute otitis media (such as mastoiditis, meningitis, facial nerve paralysis, lateral sinus thrombosis, intracranial abscess), or prior to planned hyperbaric oxygen therapy [2]. The primary underlying issue for all of the indications listed above is thought to be eustachian tube dysfunction, including acute otitis media which is thought to be a complication of upper respiratory tract infections in patients with 
underlying eustachian tube dysfunction $[3,4]$. The goal of tympanostomy tube placement is never to cure the underlying condition but to allow aeration of the middle ear until the patients' eustachian tube starts to function properly.

Children are prone to eustachian tube dysfunction for several reasons, including the small caliber of their eustachian tube, its horizontal direction, and their immature immune systems [5]. Specific subpopulations of children are at higher risk of eustachian tube dysfunction, including those with cleft palate [6], Down syndrome [7], and other children with craniofacial abnormalities [8]. These children have a significantly higher rate of tympanostomy tube insertion and tend to have more prolonged issues with eustachian tube dysfunction and often require multiple sets of tympanostomy tubes.

Although tympanostomy tube placement is considered to be a simple, low-risk intervention and is performed very commonly, there can be significant complications associated with the procedure. These include post-operative otorrhea, myringosclerosis, tympanic membrane atrophy, tympanic membrane perforation, cholesteatoma and granulation tissue [9], as well as risks associated with general anesthesia. Therefore, it is important to obtain a thorough history in these patients, consider other less invasive interventions when appropriate, and place tympanostomy tubes only when they are truly indicated.

\section{Treatment}

\section{Observation}

For many children, observation is an appropriate initial treatment option. The recent clinical practice guidelines specifically recommend against tympanostomy tube insertion in cases of short-duration OME and in children with a history of RAOM who do not have a middle ear effusion at the time of evaluation $[1 \bullet \bullet]$. These recommendations apply only to otherwise healthy children who are not at risk for or already experiencing developmental delays related to or exacerbated by their middle ear disease. The natural history of otitis media in children is very favorable with a high rate of spontaneous resolution; OME resolution following an episode of acute otitis media (AOM) is $59 \%$ by 1 month and $74 \%$ for 3 months [10]. Systematic reviews have not shown a benefit for children undergoing tympanostomy tube placement for RAOM only $[11,12]$; the patients' ability to clear the effusion spontaneously suggests favorable eustachian tube function. Following a period of observation, if children go on to develop persistent middle ear effusions (MEE), tympanostomy tubes may be offered.

Antibiotics are the mainstay of treatment for acute otitis media; although, since this is largely a self-limited disease, watchful waiting is often employed as the initial approach. Antibiotics have also been used prophylactically for children with RAOM, and although this has been shown to decrease episodes of AOM, this effect is modest and only lasts while the child is taking antibiotics; therefore, prophylactic antibiotics for RAOM are recommended against by the 2004 American Academy of Pediatrics clinical practice guidelines for otitis media [13•]. Similarly, there is no data to support the use of antibiotics to treat OME; a 2012 systematic review in the Cochrane database did not find any effect on the rate of ventilation tube insertion or any substantial improvement in hearing with antibiotic treatment of OME [14].

Intranasal steroids are occasionally used to treat patients with OME; it is thought that steroids will decrease nasal inflammation and adenoid size, 
resulting in improved eustachian tube function. Although individual studies have found this to be a successful intervention [15], larger systematic reviews have found that while OME may have quicker resolution in the short-term, no significant long-term benefit is demonstrated $[16,17]$. Additionally, a recent British study looking at cost-effectiveness concluded that treatment of OME with nasal steroids is not a cost-effective practice [18]. Therefore, the use of intranasal steroids is not recommended as a primary treatment modality for children with OME; however, it may be a useful adjunct in some patients during the period of watchful waiting.

\section{Minimally invasive interventions}

The underlying issue in most children with RAOM or OME is thought to be eustachian tube dysfunction. "Autoinflation" refers to using increased intranasal pressure to force the eustachian tube to open and may be achieved by performing a Valsalva maneuver or by using a variety of available devices, such as the Otovent or Politzer devices. Multiple studies have shown short-term effectiveness of these devices with minimal side effects associated with their use [19-21]. Although not widely accepted as a first-line treatment, a systematic review suggested that because of low cost and low risk of adverse effects, it is reasonable to consider the use of autoinflation devices while observing for natural resolution of OME [22].

\section{Surgical interventions}

\section{Myringotomy}

Myringotomy is a surgical procedure in which a small incision is made in the tympanic membrane. This allows for direct access to the middle ear space and for release of fluid in the middle ear, as well as the ability to obtain fluid for cultures if indicated. It is most commonly performed in conjunction with tympanostomy tube placement but can be performed as an isolated procedure. It is well known that myringotomy incisions heal spontaneously over a short period of time, and this has been confirmed in animal studies which show that almost all perforations are healed within 6-12 days [23]. A trial looking at myringotomy with or without tympanostomy tube insertion showed that myringotomy alone offered no benefit over myringotomy with tube insertion regarding the amount of time with effusion or the number of episodes of AOM $[24,25]$. Therefore, although myringotomy alone may be useful in specific situations, it is not recommended for children with RAOM or persistent OME. If children undergo surgical intervention for RAOM or OME, a tympanostomy tube should be placed.

\section{Myringotomy with tympanostomy tube placement}

Myringotomy with tympanostomy tube placement refers to a surgical procedure in which a small incision is made in the tympanic membrane and a pressure equalization tube is placed. This allows air exchange through the tympanic membrane and aeration of the middle ear space. It is considered the mainstay of treatment in children with RAOM or OME.

According to the recent American Academy of Otolaryngology-Head and Neck Surgery (AAO-HNS) guidelines, patients with chronic OME which has 
persisted longer than 3 months who have documented hearing difficulty should be offered bilateral myringotomy with tympanostomy tube placement (BMT). A subset of otherwise healthy children with mild hearing loss and chronic OME may have little to no benefit from BMT. In contrast, other children with more hearing difficulty may have significantly improved hearing and

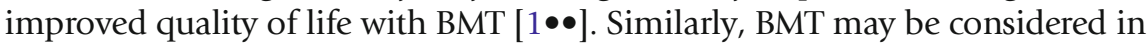
children with chronic OME who have symptoms such as behavioral problems, poor school performance, or vestibular problems which may be attributed to their OME. The final decision regarding tube placement should be made based on a conversation between the otolaryngologist and the child's caregiver, weighing the potential benefit against the risk of tympanostomy tube placement.

As discussed previously, BMT has not been shown to be a beneficial intervention in children with RAOM without evidence of persistent effusion [11, 12]. Based on these studies, the AAO-HNS guidelines recommend against BMT in children with a history of RAOM who do not have an effusion at the time of evaluation for tubes. However, in those children with RAOM who do have a middle ear effusion at the time of evaluation, the guidelines recommend offering BMT. The reasoning for this recommendation is that the presence of an effusion at the time of evaluation serves as an indication of underlying eustachian tube dysfunction and also suggests diagnostic accuracy of AOM. Of note, a recent study showed that children with RAOM with or without OME had a significantly lower quality of life scores compared to those with only OME [26], so this should be considered when making a decision regarding tympanostomy tube placement in these children. In children with RAOM, the primary rationale for placing tubes is to reduce the frequency of infections, but there are other benefits including decreased pain with ear infections and eliminating the need for systemic antibiotics. Therefore, certain subsets of children including those with severe episodes of AOM, antibiotic allergies, or other comorbid conditions may benefit from prompt tympanostomy tube placement.

The recent AAO-HNS guidelines also define "at risk children" as those children who are at increased risk for speech, language, or learning delays from either RAOM or OME due to underlying factors. The guidelines support a lower threshold for BMT in these children $[1 \bullet \bullet]$. At risk children are specifically defined as those with underlying permanent hearing loss, suspected or confirmed speech or language delay, developmental disorders, syndromes and craniofacial disorders, visual impairment, and cleft palate or developmental delay. Most physicians are aware of the increased need for tubes and increased risk of complications of OME in the cleft palate and Down syndrome populations, as both of these populations have been extensively studied [6, 7]; however, it is important to keep in mind the other, perhaps less obvious, risk factors. Therefore, it is important to get a good developmental and behavioral history and use that in conjunction with the history and physical examination when making a decision regarding tympanostomy tube placement.

The role of pre-operative audiologic testing in patients undergoing BMT placement is controversial. Some otolaryngologists routinely obtain preoperative and post-operative audiograms although the risk of hearing loss due to the procedure itself is considered largely theoretical [27]. A study by Manning, et al., in 1994 identified a 1 \% incidence of previously undiagnosed sensorineural hearing loss and concluded that hearing assessment should be a 
part of the pre-operative workup for these children [28]. The recent AAO-HNS guidelines also recommend hearing testing in all patients prior to tympanostomy tube placement $[1 \bullet \bullet]$. The results from audiometric testing contribute to decisionmaking about surgery and prompt further questions about the impact of hearing loss on a child. They also help to establish expectation of hearing improvement after tube placement.

With the rising cost of health care, there has been a push to eliminate any unnecessary testing, and the role of pre-operative audiogram has been questioned. A study looking at 1204 tympanostomy tubes placed found a $1.3 \%$ incidence of pre-existing sensorineural hearing loss consistent with Manning's findings in 1994 and no cases of postoperative conductive or sensorineural hearing loss [27]; this suggests that routine pre-operative testing may not be necessary. Regardless of the decision to obtain pre-operative audiometric data, it is generally accepted that patients should have post-operative audiograms-either as a baseline hearing assessment in patients who did not have a preoperative audiogram or as a post-op comparison in those who did have a pre-operative audiogram $[27,28]$.

Parents should be counseled that the goal of the tubes is to provide middle ear aeration but that they will not fix the eustachian tube dysfunction. Although the presence of tubes completely eliminates middle ear infections in some children, some children continue to have middle ear infections, which present with purulent otorrhea and are treated with ototopical drops. It is also important that the caregiver understands that there is no guarantee on how long the tubes will stay in place, some extrude prematurely and some remain in place too long, requiring surgical removal, but the majority extrude within 1-2 years. $[1 \bullet \bullet]$ In some children, eustachian tube function has normalized by the time the tubes have extruded; however, up to $50 \%$ of children require reinsertion of tympanostomy tubes [29]. Following tube insertion, children should follow up intermittently with their otolaryngologist until the tubes have extruded. They should also be seen following tube extrusion to evaluate the status of the middle ear and eardrum and determine whether any further intervention is required, such as reinsertion of tubes or repair of an eardrum perforation. Recommendations for tympanostomy tube placement are summarized in Table 1.

Table 1. Recommendations for tympanostomy tube placement in children

\begin{tabular}{|c|c|}
\hline Scenario & Recommendation \\
\hline OME $<3$ months & Observe \\
\hline OME $>3$ months, asymptomatic & Optional BMT \\
\hline OME $>3$ months, symptomatic & BMT \\
\hline RAOM with MEE & BMT \\
\hline RAOM without MEE & Consider BMT if impacting QOL \\
\hline At risk children ${ }^{\text {a }}$ with RAOM or OME of any duration & BMT \\
\hline
\end{tabular}




\title{
Adenoidectomy
}

\section{Conclusions}

\begin{abstract}
Adenoidectomy has been described either with or without tympanostomy tube placement as a treatment for OME. Historically, it was thought that large adenoids may mechanically obstruct the eustachian tube orifice, thus resulting in eustachian tube dysfunction and OM. Multiple recent studies suggest that in fact it is the formation of biofilms on the adenoid bed which contribute to OME rather than the size of the adenoids [30, 31]. Adenoidectomy is a more invasive surgical procedure than BMT alone, requiring intubation and increased time under general anesthesia as well as a risk of bleeding and increased postoperative pain. Adenoidectomy with BMT has been shown to result in a decreased need for repeat BMT in children over 4; however, this effect is not present in younger children. ${ }^{31}$ Most studies agree that there is limited benefit of adenoidectomy in children younger than 2-3 years of age [32] but that children over the age of 4 with persistent OME may benefit from adenoidectomy $[33 \bullet \bullet, 34]$ with BMT compared to BMT alone. Practice patterns vary, but it is reasonable to consider adenoidectomy with BMT in children over the age of 4 with persistent $\mathrm{OME}$, in those who require multiple sets of tubes, or in those with concurrent significant nasal obstruction or sleep apnea.
\end{abstract}

The recent AAO-HNS guidelines for tympanostomy tube placement provide an excellent framework for decisionmaking in children with RAOM and chronic OME. Children with RAOM who have an effusion at the time of evaluation should be offered tympanostomy tube placement; those who do not have an effusion at the time of evaluation can undergo observation with consideration of tympanostomy tube placement if the RAOM is significantly impacting their quality of life. The majority of otherwise healthy children with OME should undergo an initial period of observation for up to 3-6 months for spontaneous resolution of the effusion prior to considering tympanostomy tube placement. However, in children who are at increase risk of developmental delays, such as those with various syndromes or craniofacial abnormalities or children who are already expressing delays as a result of the effusion, surgical intervention should be performed more promptly. Although generally a low-risk procedure, caregivers should be aware of post-operative considerations and potential complications prior to their child undergoing tympanostomy tube placement.

\section{Compliance with Ethics Guidelines}

\section{Conflict of Interest}

Bianca Siegel and David H. Chi declare that they have no conflict of interest.

\section{Human and Animal Rights and Informed Consent}

This article does not contain any studies with human or animal subjects performed by any of the authors. 


\section{References and Recommended Reading}

Papers of particular interest, published recently, have been highlighted as:

- Of importance

-• Of major importance

1.• Rosenfeld RM, Schwartz SR, Pynnonen MA, Tunkel DE, Hussey HM, Fichera JS. Clinical practice guideline: tympanostomy tubes in children. Otolaryngol Head Neck Surg. 2013;149(IS):S1-35.

This paper outlines clinical practice guidelines from the American Academy of Otolaryngology. All practicing otolaryngologists who perform tympanostomy tube insertion should be aware of these guidelines.

2. Handler SD. Current indications for tympanostomy tubes. Am J Otolaryngol. 1994;15(2):103-8.

3. Harmes KM, Blackwood A, Burrows HL, Cooke JM, Van Harrison R, Passamani PP. Otitis media: diagnosis and treatment. Am Fam Phys. 2013;88(7):435-40.

4. Pelton SI, Liebovitz E. Recent advances in otitis media. Pediatr Infect Dis J. 2009;10(Suppl):S133-7.

5. Corbeel L. What is new in otitis media? Eur J Pediatr. 2007;166:511-9.

6. Kuo CL, Tsao YH, Cheng HM, Lien CF, Hsu CH, Huang $\mathrm{CY}$, et al. Grommets for otitis medial with effusion in children with cleft palate: a systematic review. Pediatrics. 2014;134(5):983-94.

7. Paulson LM, Weaver TS, Macarthur CJ. Outcomes of tympanostomy tube placement in children with Down syndrome-a retrospective review. Int J Pediatri Otorhinolaryngol. 2014;78:223-6.

8. Di Francesco R, Paulucci B, Nery C, Bento RF. Craniofacial morphology and otitis media with effusion in children. Int J Pediatr Otorhinolaryngol. 2008;72(8):1151-8.

9. Vlastarakos PV, Nikolopoulos TP, Korres S, Tavoulari E, Tzagaroulakis A, Ferekidis E. Grommets in otitis media with effusion: the most frequent operating in children. Eur J Pediatr. 2007;166(5):385-91.

10. Rosenfeld RM, Kay D. Natural history of untreated otitis media. Laryngoscope. 2003;113(10):1645-57.

11. Hellstrom S, Groth A, Jorgensen F, Pettersson A, Ryding M, Uhlen I, et al. Ventilation tube treatment: a systematic review of the literature. Otolaryngol Head Neck Surg. 2011;145(3):383-95.

12. Damoisoaux RAJM, Rovers MM. AOM in children. Clin Evid. 2011;05:301.

13. Lieberthal AS, Carroll AE, Chonmaitree T, Ganiats TG, Hoberman A, Jackson MA, et al. Clinical practice guideline: the diagnosis and management of acute otitis media. Pediatrics. 2013;131(3):e964-99.

These clinical practice guidelines are from the American Academy of Pediatrics. Management of acute otitis media and appropriate timing of referral to an otolaryngologist are discussed.

14. van Zon A, van der Heijden GJ, van Dongen TM, Burton MJ, Schilder AG. Antibiotics for otitis media with effusion in children. Cochrane Database Syst Rev. 2012;9, CD009163.

15. Cengel S, Akyol MU. The role of topical nasal steroids in the treatment of children with otitis media with effusion and/or adenoid hypertrophy. Int J Pediatr Otorhinolaryngol. 2006;70(4):639-45.

16. Thomas CL, Simpson S, Butler CC, van der Voort JH. Oral or topical nasal steroids for hearing loss associated with otitis media with effusion in children. Cochrane Database Syst Rev. 2006;3, CD001935.

17. Simpson SA, Lewis R, van der Voort J, Butler CC. Oral or topical nasal steroids for hearing loss associated with otitis media with effusion in children. Cochrane Database Syst Rev. 2011;5, CD001935.

18. Petrou S, Dakin H, Abangma G, Benge S, Williamson I. Cost-utility analysis of topical intranasal steroids for otitis media with effusion based on evidence from the GNOME trial. Value Healthy. 2010;13(5):543-51.

19. Bidarian-Miniri A, Ramos MJ, Goncalves I, Ejnell H. A new device for treatment of persistent otitis media with effusion. Int J Pediatr Otorhinolaryngol.

2013;77:2063-70

20. Blanshard JD, Maw AR, Bawden R. Conservative treatment of otitis media with effusion by autoinflation of the middle ear. Clin Otolaryngol Allied Sci. 1993;18(3):188-92.

21. Silvola J, Kivekas I, Poe DS. Balloon dilation of the cartilaginous portion of the eustachian tube. Otolaryngol Head Neck Surg. 2014;151(1):125-30.

22. Perera R, Glasziou PP, Heneghan CJ, McLellan J, Williamson I. Autoinflation for hearing loss associated with otitis media with effusion. Cochrane Database Syst Rev. 2013;5, CD006285.

23. Tahar A, Hultcrantz M. Healing of laser-induced tympanic membrane perforations in rats: no contribution of granulocyte colony-stimulating factor or Gelfoam. Int J Pediatr Otorhinolaryngol. 2012;76(7):963-8.

24. Mandel EM, Rockette HE, Bluestone CD, Paradise JL, Nozza RJ. Efficacy of myringotomy with and without tympanostomy tubes for chronic otitis media with effusion. Pediatr Infect Dis J. 1992;11(4):270-7.

25. Mandel EM, Rockette HE, Bluestone CD, Paradise JL, Nozza RJ. Myringotomy with and without tympanostomy tubes for chronic otitis media with effusion. Arch Otolaryngol Head Neck Surg. 1989;115(10):1217-24.

26. Heidemann $\mathrm{CH}$, Lauridsen $\mathrm{HH}$, Kjeldsen $\mathrm{AD}$, Faber $\mathrm{CE}$, Johansen EC, Godballe C. Quality-of-life differences among diagnostic subgroups of children receiving ventilating tubes for otitis media. Otolaryngol Head Neck Surg 2015: epub ahead of print. 
27. Emery M, Weber PC. Hearing loss due to myringotomy and tube placement and the role of preoperative audiograms. Arch Otolaryngol Head Neck Surg. 1998;124(4):421-4.

28. Manning SC, Brown OE, Roland PS, Philips DL. Incidence of sensorineural hearing loss in patients evaluated for tympanostomy tubes. Arch Otolaryngol Head Neck Surg. 1994;120(8):881-4.

29. Mandel EM, Swarts JD, Casselbrant ML, Tekely KK, Richert BC, Seroky JT, et al. Eustachian tube function as a predictor of the recurrence of middle-ear effusion in children. Laryngoscope. 2013;123(9):2285-90.

30. Park K. Otitis media and tonsils-role of adenoidectomy in the treatment of chronic otitis media with effusion. Adv Otorhinolaryngol. 2011;72:160-3.

31. Saafan ME, Ibrahim WS, Tomoum MO. Role of adenoid biofilm in chronic otitis media with effusion in children. Eur Arch Otorhinolaryngol.

2013;270(9):2417-25.
32. Casselbrant ML, Mandel EM, Rockette HE, Kurs-Lasky $\mathrm{M}$, Fall PA, Bluestone CD. Adenoidectomy for otitis media with effusion in 2-3-year-old children. Int J Pediatr Otorhinolaryngol. 2009;73(12):1718-24.

33.• Mikals SJ, Brigger MT. Adenoidectomy as an adjuvant to primary tympanostomy tube placement: a systematic review and meta-analysis. JAMA Otolaryngol Head Neck Surg. 2014;140(2):95-101.

Adenoidectomy is commonly performed as an adjuvant at the time of secondary tympanostomy tube placement; however, this paper looks at adenoidectomy at the time of primary tympanostomy tube placement. They find that in children older than 4 years of age, adenoidectomy at the time of primary tympanostomy tube placement may decrease the need for repeat surgery.

34. Boonacker CW, Rovers MM, Browning GG, Hoes AW, Schilder AG, Burton MJ. Adenoidectomy with or without grommets for children with otitis media: an individual patient data meta-analysis. Health Technol Assess. 2014;18(5):1-118. 